\title{
Conclusions du Programme National de Recherche PNR-31 «Changements climatiques et Catastrophes naturelles» : Impacts sur la société en Suisse, pistes pour le futur...
}

\section{Pierre Kunz, Genève}

\section{Introduction}

A l'issue de six années de recherche, le PNR 31 s'est conclu en 1998. Selon le mandat qui lui a été conféré par le Fonds National suisse, le PNR 31 a eu comme objectif une meilleure connaissance des processus climatiques et météorologiques conduisant aux catastrophes naturelles sur le territoire helvétique. L'évolution attendue à moyen terme des températures atmosphériques $\left(+1.8^{\circ}\right.$ à $+2^{\circ} \mathrm{C}$ pour l'horizon 2030) selon les scénarios climatiques globaux appliqués à l'échelle alpine (IPCC 1996, BENISTON et al. 1994, OHMURA et al. 1996), a servi de base de travail à ce programme. Cet article présente les principaux résultats et conclusions formulées pour les domaines concernant spécifiquement les dangers naturels et la société en Suisse (BADER \& KunZ 1998).

\section{L'impact climatique sur les dangers naturels}

Les dangers naturels étudiés dans le cadre du PNR 31 à travers de nombreux exemples dans les Alpes et le Plateau, sont les suivants (Fig. 1 et KunZ 1996) :

- les crues, les inondations, les coulées boueuses et les laves torrentielles

- les instabilités de terrain (glissements, chutes de blocs, éboulements, écroulements)

- les avalanches et les ruptures de glaciers

- les tempêtes (hivernales, foehn et grêle)

- les incendies de forêt

- les tremblements de terre (contribution au zonage sismique).

Les régions de montagne se révèlent être très sensibles aux modifications climatiques et environnementales, elles sont aussi le siège d'intenses activités humaines. Les zones périglaciaires au-dessus de $2400 \mathrm{~m}$ représentent de bons témoins des changements du climat : le réchauffement atmosphérique accentuera encore le recul des glaciers et la remontée en altitude du pergélisol, dès lors il faudra s'attendre à une érosion accélérée des pentes constituées de matériaux mal consolidés, anciennement gelées ou recouvertes par des glaciers (HAEBERLI et al. 1999). Au cours des 100 dernières années, une remontée de la limite inférieure du pergélisol de 150 à $250 \mathrm{~m}$ a été relevée, ainsi qu'un recul important des gla- ciers (depuis 1850 : perte de $27 \%$ des surfaces et de $33 \%$ de volume des glaciers originels, MAISCH et al. 1999). Les scénarios pour le siècle prochain prévoient encore une accélération du processus de fonte (horizon 2100 : plus de $50 \%$ des glaciers actuels auront disparus !) et une remontée supplémentaire du pergélisol de 200 à $700 \mathrm{~m}$. La fonte des glaces impliquera non seulement une modification des paysages, mais également un danger accru pour les constructions érigées sur le pergélisol (téléphériques, stations d'altitude). L'activité des avalanches, elle, ne semble pas s'être modifiée avec le réchauffement du climat, toutefois les mesures de protection entreprises dès 1970 semblent avoir contribué à ce statu quo (SCHNEEbeli et al. 1998).

Les événements météorologiques extrêmes comme les fortes précipitations et les tempêtes se révèlent prédominants comme facteurs déclenchants de catastrophes naturelles de type hydrologiques : crues, inondations et laves torrentielles (KUNZ 1996). Les périodes météorologiques «critiques» conduisant à une accélération du cycle de l'eau correspondent à la fin de l'été, à l'automne et surtout à l'hiver, lorsque le réchauffement est associé à une remontée de l'isotherme $0^{\circ} \mathrm{C}$. Une modification des écoulements est attendue, avec passage d'un régime nival vers un régime pluvial au semestre d'hiver. Des crues caractérisées par de forts débits, avec des charges élevées en matériaux solides, seront probablement plus fréquentes. Une croissance puis une vidange brutale de lacs péri- et sous-glaciaires en altitude, ainsi que le déclenchement de coulées boueuses et de laves torrentielles sans parallèles historiques, sont attendus à toutes les zones d'altitude. Au semestre d'été par contre, on assistera à une réduction des écoulements, avec des périodes d'étiage plus marquées.

En ce qui concerne les instabilités de terrain, l'impact des changements climatiques se marquera dans l'accélération de l'érosion des sols, des pentes et du lit des écoulements, une modification de la stabilité des glaciers suspendus (par fracturation interne), ainsi que des dangers accrus de chutes de blocs et de ruptures de falaises rocheuses par circulations internes d'eau sont prévisibles. Enfin, une recrudescence ou une réactivation probables des glissements de terrain, en particulier sur du flysch et des terrains meubles, est attendue dans des zones préalpines situées à basse altitude $(<1500 \mathrm{~m})$. Des événements paroxysmiques du type de celui de Falli-Hölli/FR en 1994 ne sont pas exclus (LATELTin et al. 1998). 


\section{L'impact climatique sur l'économie}

Outre les catastrophes naturelles, l'impact des modifications du climat sur notre société est essentielle et intéresse à priori une vaste palette d'utilisateurs. Trois régions alpines d'étude ont fait l'objet d'une analyse ciblée dans le cadre du PNR 31, elles ont permis de transmettre et de confronter les résultats du PNR 31 avec les autorités et les populations locales : la Surselva (Grisons), les Vallées de la Viège (Valais) et la Vallée des Ormonts (Vaud). Dans ces régions, les aspects touchant l'aménagement du territoire, les dangers naturels, l'économie locale (tourisme et agriculture), enfin la perception des risques ont été plus spécialement abordés (Fig. 2).

Il apparaît qu'un changement de la probabilité d'occurrence des dangers naturels, conduira à une recrudescence des dommages consécutifs. Ceci concerne en premier lieu : le domaine touristique (en particulier le tourisme hivernal), les événements de crues et d'inondations (impacts sur les infrastructures et l'habitat), enfin l'économie (agriculture et production électrique). Les coûts annuels supplémentaires ont été estimés globalement à 2300 à 3200 millions de francs, ce qui correspond à environ $1 \%$ du produit national brut de l'année 1995 (Meier 1998).

Le domaine économique lié au tourisme hivernal en Suisse (ski), devrait subir globalement à l'avenir des pertes dues au réchauffement. Les régions préalpines et jurassiennes seront plus particulièrement touchées contrairement aux régions alpines plutôt favorisées. Une élévation en altitude de la limite d'enneigement assuré, passant des $1200 \mathrm{~m}$ actuels à $1500 \mathrm{~m}$ à l'avenir (horizon 2050), conduira à une nouvelle répartition des domaines skiables : les régions «gagnantes» situées au-dessus de 1200-1500m continueront à bénéficier de conditions d'enneigement relativement sûres, les régions «perdantes ou défavorisées» situées à plus basse altitude, verront leur offre touristique d'hiver se réduire fortement (ABEGG 1996).

Au sujet du domaine agricole, la productivité devrait se trouver réduite à cause de la réduction des précipitations estivales. Toutefois, l'effet de fertilisation du $\mathrm{CO}_{2}$ atmosphérique favorisera le développement de la végétation, cet effet permettra ainsi de compenser ou de surpasser les pertes de production. En termes de rendements agricoles, les changements climatiques vont favoriser la position des plaines au dépend de l'agriculture de montagne, en raison principalement de la capacité de concurrence plus réduite de celle-ci face aux exploitations du Plateau (ex : fourrage). Les écarts de revenus risquent à l'avenir encore de s'accroître entre ces régions (FLÜCKIGER \& RIEDER 1997).

\section{L'impact climatique sur la société : problèmes de perception}

La perception face aux risques environnementaux et climatiques est liée à l'appartenance à différents milieux sociaux (privé, politique ou économique) et à différentes cultures $d u$ risque (individualiste, hiérarchique ou égalitaire). Cette perception est aussi indissociable du contexte socio-économique actuel, où les paramètres de solidarité, de difficultés économiques ou encore de changements structurels interviennent.

Le réchauffement global ne semble pas constituer une préoccupation majeure des populations, en particulier de montagne, la priorité est clairement donnée aux problèmes locaux plus cruciaux. Parmi les soucis actuels des autorités locales approchées, figurent : la sécurité et l'entretien de l'habitat, des voies de communication et des équipements face aux dangers naturels actuels, l'avenir des activités touristiques (en particulier le ski), le maintien des activités économiques : agriculture de montagne et places de travail (dans les industries locales comme à St-Nicolas/VS), l'évolution de l'utilisation du territoire (abandon des surfaces entretenues, extension de la forêt) ou encore les futures ressources en eau (STUCKI \& RognON 1998 et Fig. 2).

Un exemple caractéristique est illustré par le manque d'enneigement ayant affecté tous les domaines skiables de Suisse à la fin des années 1980. Cette situation délicate, voire catastrophique selon les régions (cf. Préalpes), a donné lieu à une réflexion chez les responsables du tourisme sur les alternatives possibles à la pénurie de neige ou les compléments à l'offre du tourisme hivernal «classique». Ces idées sont prometteuses et témoignent déjà d'un effort d'adaptation des professionnels du tourisme, bien qu'il ne faille pas en attendre trop car à l'heure actuelle il n'existe aucune solution réaliste pouvant remplacer le tourisme du ski. Ces responsables peuvent dès lors susciter et encourager des propositions de diversifications des activités touristiques, indépendantes de la présence de neige, lors de chaque investissement (ABEGG 1996).

\section{Connaissances et stratégies traditionnelles des risques}

Dans les trois régions d'étude du PNR 31, il est clairement apparu une connaissance traditionnelle des risques naturels par les populations résidentes (de montagne). Chez les personnes interrogées, l'expérience et l'observation personnelles sont prédominantes pour la représentation des dangers naturels, où l'espace vécu revêt toute son importance. Les événements fréquents observables sont en général bien connus, comme les avalanches. Par contre, la perception des événements épisodiques - rares ou exceptionnels - comme les instabilités de terrain, les éboulements et les crues, est nette- 


\begin{tabular}{|c|c|c|c|}
\hline Secteurs & $\begin{array}{l}\text { Probabilité } \\
\text { d'occurrence }\end{array}$ & $\begin{array}{l}\text { Etendue des } \\
\text { impacts }\end{array}$ & Implications \\
\hline $\begin{array}{l}\text { Limite inférieure de la neige } \\
+300 \text { à }+500 \mathrm{~m} \\
\text { Durée d'enneigement }-20 \text { à }-40 \%\end{array}$ & $\begin{array}{l}+++ \\
++\end{array}$ & $\begin{array}{l}--- \\
---\end{array}$ & $\begin{array}{l}\text { - Préalpes, Jura. } \\
\text { - Tourisme, surtout tourisme } \\
\text { hivernal. }\end{array}$ \\
\hline $\begin{array}{l}\text { Nombre de glaciers }-80 \% \\
\text { Surfaces glaciaires }-70 \%\end{array}$ & $\begin{array}{l}+++ \\
+++\end{array}$ & $\begin{array}{l}- \\
-\end{array}$ & $\begin{array}{l}\text { Tourisme, environnement, } \\
\text { production électrique, société, } \\
\text { ressources en eau. }\end{array}$ \\
\hline $\begin{array}{l}\text { Limite inférieure du pergélisol } \\
+200 \text { à }+700 \mathrm{~m}\end{array}$ & +++ & --- & $\begin{array}{l}\text { - Haute montagne (>2400 m). } \\
\text { - Infrastructures, téléphériques, } \\
\text { éventuellement habitat. }\end{array}$ \\
\hline $\begin{array}{l}\text { Modification de } \\
\text { l'activité des avalanches }\end{array}$ & - & $( \pm)$ & $\begin{array}{l}\text { - Personnes, bâtiments, } \\
\text { biens, infrastructures. }\end{array}$ \\
\hline $\begin{array}{l}\text { Coulées boueuses alpines } \\
\text { Coulées boueuses péri-alpines }\end{array}$ & $\begin{array}{l}+ \\
\pm\end{array}$ & $\begin{array}{l}- \\
-\end{array}$ & $\begin{array}{l}\text { - Bâtiments, biens, } \\
\text { infrastructures, paysages. }\end{array}$ \\
\hline $\begin{array}{l}\text { Régime hydrologique : } \\
\text { plus d'écoulements hivernaux }\end{array}$ & ++ & - & $\begin{array}{l}\text { - Environnement, ressources } \\
\text { en eau, production électrique. }\end{array}$ \\
\hline $\begin{array}{l}\text { Crues (surtout alpines) } \\
\text { Inondations }\end{array}$ & $\begin{array}{l}+ \\
++\end{array}$ & $\begin{array}{l}--- \\
---\end{array}$ & $\begin{array}{l}\text { - Personnes, bâtiments, } \\
\text { biens, infrastructures. }\end{array}$ \\
\hline $\begin{array}{l}\text { Systèmes aquifères } \\
\text { Sécheresse }\end{array}$ & $\begin{array}{l}+ \\
++\end{array}$ & $\begin{array}{l}- \\
-\end{array}$ & $\begin{array}{l}\text { - Ressources en eau, approvision- } \\
\text { nement, agriculture. }\end{array}$ \\
\hline $\begin{array}{l}\text { Tempêtes estivales / Grêle } \\
\text { Tempêtes hivernales }\end{array}$ & $\begin{array}{l}++ \\
+\end{array}$ & $\begin{array}{l}- \\
--\end{array}$ & $\begin{array}{l}\text { - Bâtiments, biens, infrastructures, } \\
\text { économies agricole et forestière. }\end{array}$ \\
\hline $\begin{array}{l}\text { Forêts } \\
\text { - non adaptées à leur milieu : } \\
25-30 \%, \text { plus 5-30\% futurs } \\
\text { - Accroissement de la biomasse } \\
\text { - Limite supérieure }+100 \text { à }+300 \mathrm{~m} \\
\text { - Incendies au Sud des Alpes }\end{array}$ & $\begin{array}{l}++ \\
\pm \\
+ \\
++\end{array}$ & $\begin{array}{l}- \\
(+) \\
+ \\
-\end{array}$ & $\begin{array}{l}\text { - Baisse de productivité. } \\
\text { - Augmentation de productivité. } \\
\text { - Augmentation des fonctions } \\
\text { protectrices. } \\
\text { - Habitat, baisse de productivité. }\end{array}$ \\
\hline Economie rurale (cultures) & ++ & \pm & - Productivité agricole, revenus. \\
\hline Emigration, Réfugiés & +++ & --- & - Société, économie. \\
\hline
\end{tabular}

Fig. 1 : Vue d'ensemble des impacts des changements climatiques pour la Suisse :

horizon 2030 - 2050 (MEIER 1998)

Überblick über die Folgen der Klimänderungen für die Schweiz: Zeithorizont 2030 - 2050

Global view of the impacts of climate changes for Switzerland: time-scale $2030-2050$ 


\author{
Légende / Legende / Legend \\ Probabilité d'occurrence des impacts des changements climatiques (scénarios) : \\ Eintretenswahrscheinlichkeit von Wirkungen aufgrund der Klimaänderungen (Szenarien): \\ Probability of occurrence of the impacts of climate changes (scenarios): \\ +++ (Relativement) forte probabilité d'occurrence / (Relativ) hohe Eintretenswahrscheinlichkeit / \\ (Relatively) High probability of occurrence \\ $+\quad$ Probabilité moyenne d'occurrence / Mittlere Eintretenswahrscheinlichkeit / \\ Medium probability of occurrence \\ $+\quad$ Faible probabilité d'occurrence / Geringe Eintretenswahrscheinlichkeit / Low probability of occurrence \\ $\pm \quad$ Probabilité d'occurrence nulle ou très incertaine / Keine bzw. sehr ungewisse Eintretenswahrscheinlichkeit \\ / Probability of occurrence: zero or very uncertain \\ - $\quad$ Occurrence improbable / Abnahme der Eintretenswahrscheinlichkeit / Improbable occurrence \\ Etendue des impacts potentiels (dommages, respectivement profits) : \\ Ausmass der potentiellen Auswirkungen (Schaden- bzw. Nutzenpotential): \\ Extent of potential impacts (damages, respectively profits): \\ - - - Impacts négatifs importants : potentiel élevé de dommages / Bedeutende Auswirkungen mit negativen \\ Folgen: hohes Schadenpotential / Important negative impacts: high potential of damages \\ - - Impacts négatifs moyens : potentiel moyen de dommages / Mittlere Auswirkungen mit negativen Folgen: \\ mittleres Schadenpotential / Medium negative impacts: middle potential of damages \\ - $\quad$ Impacts réduits avec potentiel limité de dommages / Geringe Auswirkungen mit geringem \\ Schadenpotential / Low impacts with limited potential of damages \\ $+\quad$ Faibles impacts positifs : potentiel de profits / Geringe Auswirkungen mit positiven Wirkungen: \\ Nutzen- bzw. Gewinnpotential / Weak positive impacts: potential of profits \\ ( ) Impacts très incertains / Sehr unsichere Auswirkungen / Very uncertain impacts
}

ment plus lacunaire (exemple les Ormonts/VD, Schoeneich \& Busset-Henchoz 1998). Ces événements catastrophiques ne sont vraiment connus que lorsqu'ils touchent l'expérience personnelle ou lorsqu'ils occasionnent des dégâts.

L'attitude dominante correspond à une acceptation ou une accommodation aux risques naturels par les populations résidentes, sous la forme d'une «cohabitation». Il s'agit en réalité d'un «choix de société» : l'occupation du territoire en zones montagneuses ne traduit pas une méconnaissance ou une inconscience face aux risques naturels, mais révèle toute une variété de stratégies traditionnelles d'adaptation, d'évitement et de protection, relevant d'un «risque calculé» ainsi que de la nécessité et des contraintes de survie. Ces stratégies sont souvent associées à une communication traditionnelle écrite des dangers à travers des chroniques ou des archives (SChoeneich \& Busset-Henchoz 1998). Ces stratégies, souvent très anciennes, gardent aujourd'hui encore toute leur validité et mériteraient d'être mieux intégrées dans les processus de gestion et de prévention des risques naturels, en regard des mécanismes incitatifs de partage des coûts, du subventionnement ou des assurances. On peut citer des adaptations d'ordre constructif (digues, renforts, etc.), planifié (positionnement des villages, modes d'exploitation des terres) ou encore comportemental (déplacements selon les conditions météorologiques ou saisonnières). Des zones à risques ont par exemple été occupées et exploitées depuis des siècles, par nécessité agricole surtout (Herold-Revaz \& Dulex Putallaz 1998).

\section{Besoin d'informations}

Les changements climatiques représentent en fait une problématique nouvelle préoccupante surtout pour la nouvelle génération, pour les personnes plus âgées le climat constituant par nature un facteur constant. L'intérêt et l'inquiétude des populations deviennent en fait croissants, lorsque celles-ci prennent conscience des modifications possibles de leur environnement physique direct.

A ce stade, un renforcement de la perception et de la prise de conscience des risques climatiques, en particulier en direction des résidents secondaires et des touristes, apparaît nécessaire en vue d'une meilleure responsabilisation des individus (Herold-Revaz \& Dulex Putallaz 1998). D'avantages d'actions de sensibilisation de la population, incorporant une prise en compte des incertitudes actuelles, doivent pouvoir améliorer la compréhension du système climatique, de son évolution et de ses conséquences face aux dangers naturels. Cette sensibilisation permettra dès lors de conserver les informations face aux «pertes de mémoire» des populations. La mémoire représente une clé pour le futur, mais de nos jours elle ne franchit pas la période d'une génération (30 ans), une perte de signification des événements est liée en particulier à la mobilité accrue des individus. La transmission d'une information précoce, systématique, claire, compréhensible et sereine (surtout pas alarmiste ni catastrophiste) des événements naturels et de leur évolution, représente une tâche primordiale des spécialistes scientifiques, mais aussi des administrati- 
ons et des médias, à l'adresse des autorités responsables et des populations (CEDOS 1998). Les populations concernées réagissent effectivement aux informations d'ordre technique/scientifique qui leur sont utiles et non au sensationnalisme.

\section{7 «Culture de la Prévention»}

Contrairement aux générations passées - rurales -, l'acceptabilité générale face aux risques naturels diminue dans notre société actuelle - plus urbaine -. Alors que la vulnérabilité des biens exposés continue de s'accroître, les coûts des dégâts par événement catastrophique augmentent, enfin les moyens financiers des collectivités pour les mesures de protection vont en se réduisant.

Un besoin d'action face aux dangers naturels s'avère nécessaire, que ce soit avec ou sans changements climatiques. En effet, comme il est clairement impossible d'intervenir partout systématiquement, une politique de protection permettant de prévenir les dommages doit être conduite. Les conditions-cadres et pressions d'ordre socio-économique, l'utilisation intensive des sols, la définition de zones appropriées à la construction (cf. tourisme) et nécessitant une haute sécurité à long

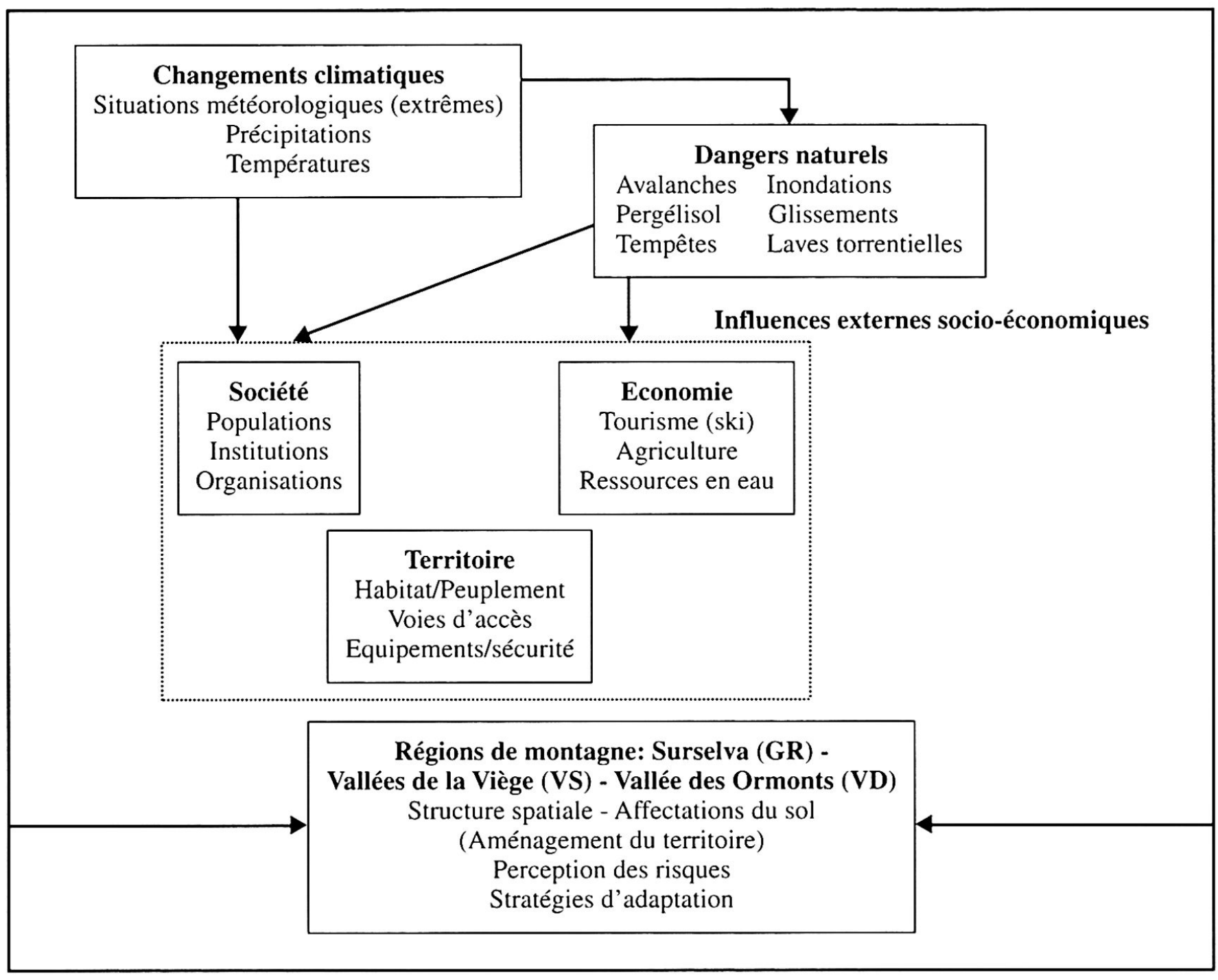

Fig. 2 : Evaluation des impacts des changements climatiques sur le développement de la société, de l'économie et des dangers naturels, sur la base d'exemples géographiques alpins (Surselva/GR, Vallées de la Viège/VS et Vallée des Ormonts/VD) (BLOETZER et al. 1998) Evaluation der Auswirkungen der Klimänderungen auf der Gesellschafts-, der Wirtschafts- und der Naturgefahrenentwicklung, anhand ausgewählter Beispiele in den Alpen (Surselva/GR, Vispertäler/VS und Vallée des Ormonts/ $V D)$

Evaluation of the impacts of climate changes on the development of the society, the economy and the natural disasters, based on geographic examples in the Alps (Surselva/GR, Vallées de la Viège/VS and Vallée des Ormonts/VD) 
terme, enfin une planification prévoyante face au potentiel futur de catastrophes naturelles, impliquent une prise en compte plus poussée des dangers naturels. Dans le cadre de l'aménagement du territoire, l'instrument des plans directeurs cantonaux paraît être le plus approprié pour cette tâche (BLOETZER et al. 1998).

L'expérience des dangers, associée aux stratégies traditionnelles et différenciées d'adaptation dans les régions de montagne, doit trouver un impact au niveau d'une politique de prévention efficace des catastrophes naturelles et être intégrées dans les processus de décision. En effet, à cause d'une pression accrue socio-économique pour une utilisation intensive des sols, avec intervention dans des zones sensibles ou des territoires potentiellement menacés par les dangers naturels, il s'avère nécessaire de soutenir une «planification prévoyante», prenant aussi en compte les connaissances actuelles sur le développement des changements environnementaux (par exemple les dangers naturels liés au climat).

\section{Pistes pour le futur...}

Les zones sujettes aux catastrophes naturelles sont déjà connues et souvent bien délimitées géographiquement grâce à des évidences géomorphologiques et historiques d'anciens événements (traces passées de crues et de laves torrentielles, signes d'anciennes instabilités, zones d'avalanches). Les zones périglaciaires et de glaciers dans les Alpes, ainsi que les zones déjà affectées dans les bordures alpines, sont à surveiller préférentiellement (ZimmermanN et al. 1997). Il est bien clair qu'il est impossible de mettre toute la Suisse sous surveillance, d'où la nécessité de cibler les zones potentiellement dangereuses. C'est pourquoi, les données historiques ou anciennes (chroniques et archives) sont essentielles pour comprendre les processus actuels et futurs (PFISTER 1998).

La connaissance approfondie des processus et des mécanismes naturels intervenants requièrent un besoin clair en informations de base et en suivi des processus (surveillance et mesures). Il faut donc soutenir l'acquisition des données et surtout ne pas oublier un facteur essentiel souvent sous-estimé : l'adaptabilité des (éco)systèmes aux phénomènes naturels et aux variations de notre environnement, par exemple des incendies ou des tempêtes peuvent accélérer le processus dynamique de rajeunissement de la forêt (cf. VIVIanE 1990). Mais les facteurs anthropiques jouent souvent un rôle prépondérant face à la variabilité élevée des processus naturels (cf. climat), comme le témoignent l'accroissement des incendies au sud des Alpes, l'extension des domaines skiables ou la pression de l'habitat. D'autre part, les mutations économiques actuelles masquent les préoccupations des décideurs et de la population face aux problèmes environnementaux.

\section{Conclusions}

Les informations acquises par le PNR 31 (BADER \& Kunz 1998) devraient placer les populations de montagne et leurs autorités devant des responsabilités nouvelles : s'informer et intégrer les connaissances actuelles en matière de changements climatiques et de prévention des catastrophes naturelles, permettant ainsi d'être à même de prendre des décisions qui vont engager les générations futures. Il est aussi important, que les acquis scientifiques soient adaptés aux conditions locales pour trouver un impact.

\section{Bibliographie}

ABEGG, B. (1996): Klimaänderung und Tourismus. Klimafolgenforschung am Beispiel des Wintertourismus in den Schweizer Alpen. - = Schlussbericht NFP 31, Zürich: 1-222.

Bader, S. \& Kunz, P. (1998): Climat et risques naturels - La Suisse en mouvement. - = Rapport scientifique final PNR 31, Genève et Zürich: 1-312.

Beniston, M., Rebetez, M., Giorgi, F. \& M.-R. MARINUCCI (1994): An analysis of regional climate change in Switzerland. - In: Theor. Appl. Climatol. 49: 135-159.

Bloetzer, W., Egli, T., Petrascheck, A., Sauter, H. \& M. STOFFEL (1998): Klimaänderungen und Naturgefahren in der Raumplanung - Methodische Ansätze und Fallbeispiele. $-=$ Synthesebericht NFP 31, Zürich: 1200.

CEDOS Communication (1998): „Le regard de Janus“, Changements climatiques et catastrophes naturelles. Ouvrage de vulgarisation grand public PNR 31. - Genève: Georg.

FlüCKIGER, S. \& P. RIEDER (1997): Klimaänderung und Landwirtschaft - Ökonomische Implikationen innerhalb der Landwirtschaft und ihres Umfeldes aus globaler, nationaler und regionaler Sicht. $-=$ Schlussbericht NFP 31, Zürich: 1-222.

Haeberli, W., Kä̈̈в, A., Hoelzle, M. Bösch, H., FunK, M., VoNder MÜHLl, D. \& F. Keller (1999): Eisschwund und Naturkatastrophen im Hochgebirge. $=$ Schlussbericht NFP 31, Zürich: 1-190.

Herold-Revaz, A., Dulex Putallaz, S. \& L. Bridel (1998): Représentations du risque d'avalanches et comportements sociaux dans deux commune valasiannes. = Rapport de travail PNR 31, Zürich: 1-165.

IPCC (1996): Climate Change 1995 - The IPCC Second Assessment Report. $-=$ Contributions of Working Group I: 1-572; Group II: 1-878; Group III: 1-448, Cambridge University Press.

KunZ, P. (1996): Dangers naturels : conséquences face au climat. - In: Programmleitung NFP 31 (Hrsg.): „Klimarisiken - Herausforderung für die Schweizer Wirtschaft". - Arbeitsbericht NFP 31, Zürich: 51-60.

Lateltin, O., Beer, C., Raetzo, H. \& C. Caron 
(1998): Instabilités de pente en terrain de flysch et changements climatiques. - = Rapport final PNR 31, Zürich: $1-168$.

Maisch, M., Wipf, A., Denneler, B., Battaglia, J. \& C. BENZ (1999): Auswirkungen von Klimaänderungen auf die Gletscher und deren Vorfelder. - = Schlussbericht NFP 31, Zürich: 1-376.

MeIER, R. (1998): Sozioökonomische Aspekte von Klimaänderungen und Natukatastrophen in der Schweiz. = Schlussbericht NFP 31, Zürich: 1-217.

Ohmura, A., Beniston, M., Rotach, M., Tschuck, P., WiLD, M. \& M.-R. MarinuCCI (1996) : Simulation of Climate Trends over the Alpine Region. Development of a physically-based modeling system for application to regional studies of current and future climates. $-=$ NRP 31 Final Scientific Report, Zürich: 1-197.

PFISTER, C. (1998): Raum-zeitliche Rekonstruktion von Witterungsanomalien und Naturkatastrophen 14961995. - = NFP 31 Schlussbericht, Zürich: 1-140.

Schneebeli, M., Laternser, M. Föhn, P. \& W. AMmANN (1998): Wechselwirkungen zwischen Klima, Lawinen und technischen Massnahmen. - = NFP 31 Schlussbericht, Zürich: 1-132.

Schoeneich, P. \& M.-C. Busset-Henchoz (1998): Les Ormonans et les Leysenouds face aux risques naturels - Représentation des risques naturels et stratégies d'occupation du territoire dans la Vallée des Ormonts (Préalpes vaudoises). - = Rapport final PNR 31, Zürich: $1-230$.

StUCKI, E. \& P. Rognon (1998): La Vallée des Ormonts face aux changements climatiques et aux catastrophes naturelles. $-=$ Rapport de synthèse PNR 31, Genève et Zürich: 1-67.

Zimmermann, M., Mani, P., Gamma, P., Gsteiger, P., Heiniger, O. \& G. HunZiKer (1997): Murganggefahr und Klimaänderung - ein GIS-basierter Ansatz. - = NFP 31 Schlussbericht, Zürich: 1-170.

\section{Résumé : Conclusions du Programme National de Recherche PNR-31 «Changements climatiques et Catastrophes naturelles» : Impacts sur la société en Suisse, pistes pour le futur...}

Le PNR 31, conclu en 1998, a eu comme objectif une meilleure connaissance des processus climatiques et météorologiques conduisant aux catastrophes naturelles en Suisse, avec en particulier une analyse détaillée des interactions entre le climat, les dangers naturels et la société. Notre pays se trouve spécialement concerné par cette thématique, en effet les régions de montagne avec leurs zones périglaciaires se révèlent être très sensibles aux modifications climatiques et environnementales, de plus elles sont aussi le siège d'intenses activités humaines.

Outre les catastrophes naturelles, l'impact des modifications climatiques sur notre société et notre économie est essentielle et intéresse à priori une vaste palette d'utilisateurs. Cependant, le réchauffement global ne semble pas constituer une préoccupation majeure des populations, la priorité étant clairement donnée aux problèmes locaux, rendus cruciaux dans le contexte socio-économique actuel difficile. Cette priorité va à l'encontre de l'attitude dominante traditionnelle, qui correspondait plutôt à une acceptation ou une accommodation aux risques naturels par les populations résidentes de montagne.

Les informations acquises par le PNR 31 devraient dès lors placer les populations et leurs autorités devant des responsabilités nouvelles : s'informer et intégrer les connaissances actuelles en matière de changements climatiques et de prévention des catastrophes naturelles, permettant ainsi d'être à même de prendre des décisions dans le sens d'une ,planification prévoyante“ des risques.

\section{Zusammenfassung: Schlussfolgerungen des Nationalen Forschungsprogramms NFP 31 «Klimaänderungen und Naturkatastrophen»: Folgen für die Gesellschaft in der Schweiz, Lehren für die Zukunft...}

Das 1998 abgeschlossene NFP 31 hatte zum Ziel, bessere Kenntnisse über die klimatischen und meteorologischen Prozesse zu gewinnen, welche in der Schweiz zu Naturkatastrophen führen können. Im speziellen sollten die Interaktionen zwischen dem Klima, den Naturgefahren und der Gesellschaft untersucht werden. Unser Land ist durch diese Thematik besonders betroffen, da sich vor allem die Bergregionen mit ihren Permafrostgebieten als sehr sensibel gegenüber Klimaschwankungen und Umweltveränderungen erweisen, dazu finden gerade hier auch intensive menschliche Aktivitäten statt.

Neben den Naturkatastrophen ist der Einfluss von Klimaschwankungen auf unsere Gesellschaft und die Wirtschaft bedeutend und für eine breite Palette von Nutzungen von Interesse. Indessen scheint die globale Erwärmung keine besondere Vorsorge bei der Bevölkerung auszulösen. Priorität haben angesichts der aktuell schwierigen sozioökonomischen Lage klar die lokalen Probleme; dies im Gegensatz zur traditionell überwiegenden Haltung der Bergbevölkerung, welche in Bezug auf die Naturgefahren eher auf ein Unterordnen oder Anpassen ausgerichtet war.

Die im Rahmen des NFP 31 gewonnenen Erkenntnisse werden die Bevölkerung und ihre Behörden vor neue Verantwortlichkeiten stellen: sich informieren und die aktuellen Kenntnisse bezüglich Klimaänderung und Katastrophenprävention in Entscheide einfliessen lassen, welche im Sinne einer «vorausschauenden Planung» unter Einbezug der Naturrisiken gefällt werden müssen. 


\section{Summary: Conclusions of the National Research Program NRP 31 «Climate Changes and Natural Disasters»: Impacts on Society in Switzerland, lessons for the future...}

The National Research Program of the Swiss National Science Foundation (NRP 31), concluded in 1998, had the objective of better understanding the climatic processes leading to natural disasters in Switzerland. In particular, detailed analyses of the interactions between the climate, the natural dangers and Society were carried out. Switzerland is especially concerned by such themes, since mountain areas with their periglacial zones prove to be very sensitive to climatic and environmental changes; moreover they are also the seat of considerable human activities.

In addition to natural disasters, the impacts of climate changes on socio-economic sectors are essential to the interests of a vast range of users. However, global warming does not seem to be a major concern for people, the priority being clearly given to local problems, which are crucial in the current socio-economic context. This is contrary to traditional dominating attitudes, which lead to the acceptance or accommodation towards natural risks by mountain communities.
The information acquired by the NRP 31 should consequently allow the general public and their authorities to face up to new responsibilities, i.e., to get information and integrate current knowledge on climate changes and the prevention of the natural disasters, thus allowing implementation of long-term risk planning.

Dr. Pierre Kunz, Direction PNR 31 «Changements climatiques et Catastrophes naturelles», Section des Sciences de la Terre, Université de Genève, 13 rue des Maraîchers, $\mathrm{CH}-1211$ Genève 4.

e-mail: pierre.kunz@terre.unige.ch 
